\title{
DOA Estimation via a Network of Dumb Sensors Under the SENMA Paradigm
}

\author{
Stefano Marano, Vincenzo Matta, Peter Willett, and Lang Tong
}

\begin{abstract}
Following the SENMA concept, we consider a wireless network of very dumb and cheap sensors, polled by a travelling “rover.' Sensors are randomly placed and isotropic: Individually, they have no ability to resolve the direction of arrival (DOA) of an acoustic wave. However, they do observe the wavefront at different times. We assume that the communication load must be as limited as possible, so that these times cannot be communicated to the rover. Notwithstanding the lack of transmission of arrival times and the lack of DOA resolution ability of the individual sensors, DOA estimation is possible and simple, and asymptotic efficiency becomes closely approximated after a reasonable number of rover snapshots. Key features are the directionality of the rover antenna, the area it surveys, and the average number of sensors inside that area, as accorded a Poisson distribution.
\end{abstract}

Index Terms-Data fusion, direction of arrival (DOA), sensor network.

\section{INTRODUCTION}

A LARGE network of extremely low-complexity (a.k.a., "dumb") sensors is employed to estimate the direction of arrival (DOA) of a plane-wave (far-field, and for concreteness, let us assume acoustic) event. The system is designed to detect the wavefront passage regardless of the signal waveform features. The sensors are isotropic: None of them has any ability at all to resolve the DOA on its own; however, each can memorize the time instant of the acoustic wavefront passage. The sensors are randomly displaced over a certain surveyed area according to a Poisson field model, as might occur were the sensors dropped by an aircraft in an unstructured way.

According to the SENMA model, a travelling rover receives (electromagnetic) signals from the sensors that lie in its field of view. As is well known, a distinct feature of sensor networks is the tradeoff between the communication load, the requirement to fuse the data, and the accuracy of the network inference goal (e.g., detection of events, parameter estimation, etc.) (e.g. see [3]). We avoid any concern about the communication burden: All of the sensors transmit to the rover using one and the same channel. The key point is that they do not transmit bits of data but simply emit an analog periodic signal made of short pulses. In aggregate, they form a train of delta-like pulses, and this is what

Manuscript received July 8, 2004; revised September 8, 2004. This work was supported in part by the Office of Naval Research. The associate editor coordinating the review of this manuscript and approving it for publication was Prof. Steven M. Kay.

S. Marano and V. Matta are with DIIIE, Università degli Studi di Salerno, I-84084 Fisciano, Italy (e-mail: marano@unisa.it; vmatta@unisa.it).

P. Willett is with the Electrical and Computer Engineering Department, University of Connecticut, Storrs, CT 06269 USA (e-mail: willett@engr.uconn.edu).

L. Tong is with the Electrical and Computer Engineering Department, Cornell University, Ithaca NY 14853 USA (e-mail: 1tong@ece.cornell.edu).

Digital Object Identifier 10.1109/LSP.2005.855543

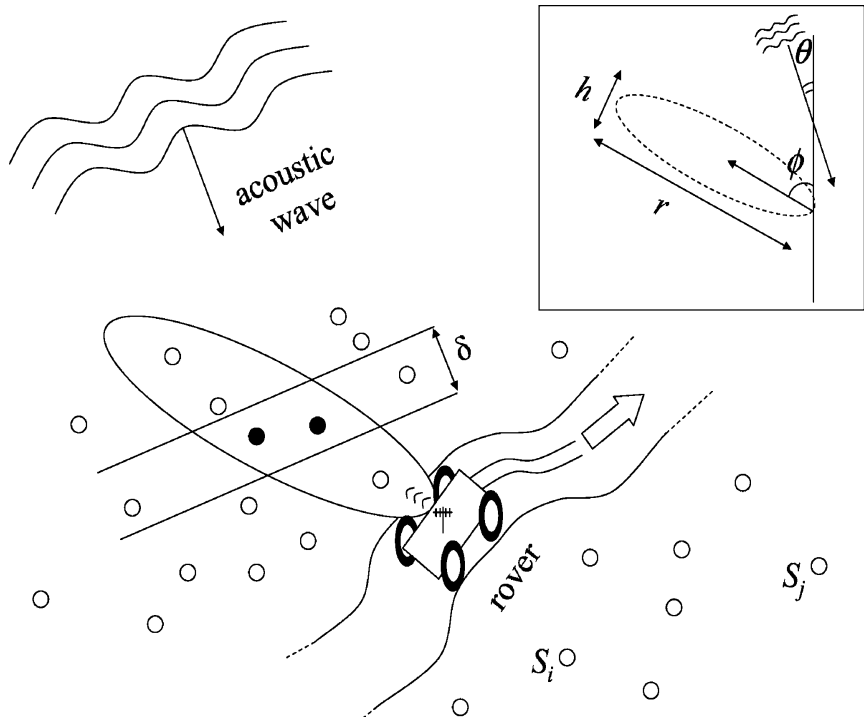

Fig. 1. Addressed scenario. A travelling rover polls the remote sensors inside its field of view. The DOA estimation procedure is based on the number of sensors that lie inside the strip of width $\delta$. The separate box introduces some notations: $\theta$ is the sought DOA, and $\phi$ is the rover orientation, whose field of view is an ellipse with axes $r$ and $h$.

the rover observes. The directionality of the rover antenna is key, and in fact, the more asymmetric is the antenna lobe, the more effective the estimation procedure becomes; ${ }^{1}$ however, there are limits, as discussed in the following.

We find it convenient to work with a reasonable, simple mathematical model: the rover's antenna pattern-probably in practice some sort of truncated cone-is modeled as an ellipse. There is no requirement for an elliptical field of view, only that the field of view is known; the ellipse makes analysis convenient and explicit.

\section{MODEL}

The notional scenario is depicted in Fig. 1. We consider a large network of wireless sensors, say, $S_{i}, i=1,2, \ldots$, covering a certain two-dimensional region. The sensors are randomly located according to a Poisson field probability model, with $\lambda$ being the sensor density per unit of area: The average number of sensors, inside an arbitrarily shaped region of area $A$, is $\lambda A$.

We assume that each $S_{i}$ is an acoustic antenna, with no directionality capabilities: Its antenna pattern is isotropic. If hit by a short-duration acoustic wavefront, coming from an arbitrary direction, sensor $S_{i}$ starts to transmit an electromagnetic periodic signal $x_{i}(t)=\sum_{k} p\left(t-\tau_{i}-k T\right)$, where $p(t)$ is a short pulse of arbitrary shape, $\tau_{i}$ is the time at which the acoustic wavefront

${ }^{1}$ The sensors are dumb and isotropic; the rover is not. 

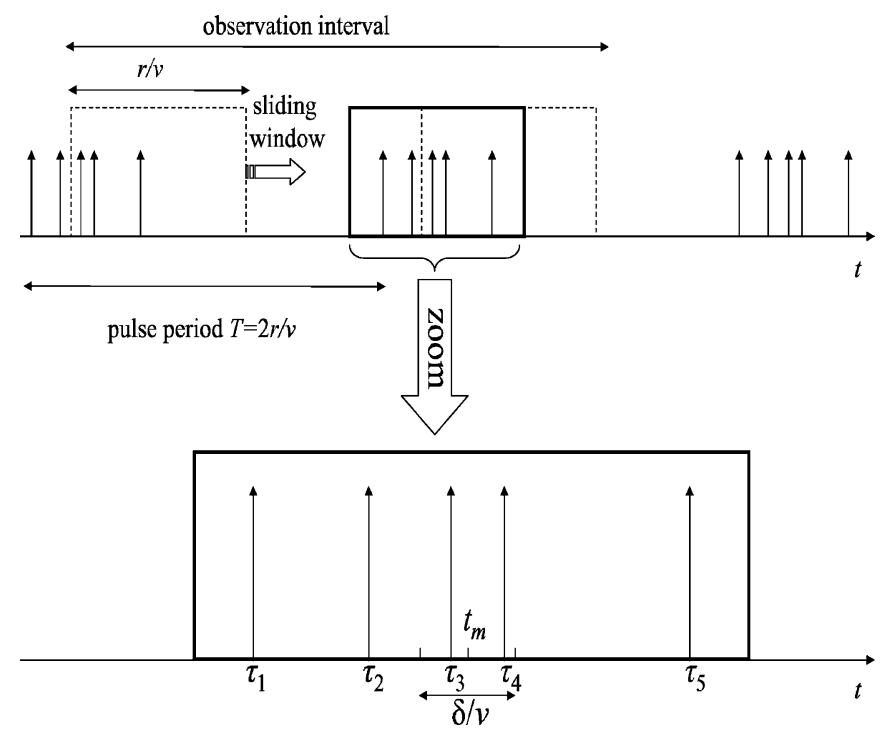

Fig. 2. Signal collected by the rover is schematically depicted in the top plot. With an appropriate observation interval (larger than twice the pulse period $T$ ), it is possible to order the pulses: They are arranged in the same order in which the sensors have emitted them (i.e., have been hit by the acoustic wavefront); see bottom plot. Here, $t_{m}$ is the middle point between $\tau_{1}$ and $\tau_{5}$, and the $\delta / v$-interval centered on $t_{m}$ includes the two pulses at $\tau_{3}$ and $\tau_{4}$. These are emitted by the two sensors inside the $\delta$-strip of Fig. 1.

impinges on the device, $T$ is an a priori chosen time interval, common to all sensors, and $k$ is an integer.

According to the SENMA paradigm [4], a roving base station (rover in Fig. 1) travels the area. For some fixed position, the rover takes a dwell: It collects, for a certain time interval, ${ }^{2}$ the signal $r(t)=\sum_{i \in \mathcal{F}} x_{i}(t)$. Such a received signal is made of the superposition of the $|\mathcal{F}|$ signals emitted by the ensemble $\mathcal{F}$ of sensors lying in its field of view. It is worth emphasizing that for reasons of analysis, one might assume that the sensors emit their pulse trains continually; but for reasons of battery life, the emissions would remain virtual until a rover requests them via a poll.

We assume that the antenna pattern is an ellipse with the main axis of length $r$ aligned to the rover and the secondary one of length $h$. It is also assumed that all signals coming from sensors inside the ellipse are visible, while conversely, none from outside can be received; this defines the rover's field of view. ${ }^{3}$

A typical waveform $r(t)$ received by the rover is schematically illustrated in Fig. 2. Note that we set $T=2 r / v$ (larger $T$ works as well), where $v$ is the speed of the acoustic wave in the medium: $r / v$ is the time needed for the acoustic wave to cover the main axis of the lobe. Such a choice enables the rover to order the observed pulses so that $\tau_{i}<\tau_{i+1}$, since sensors inside the field of view all have a maximum time interval of $r / v$ and since the pulse period is twice that. This is illustrated in Fig. 2, where the zoomed time axis (bottom) gives the correct pulse ordering. ${ }^{4}$ Clearly, the sensor positions are unknown, and the pulses are unlabeled: The rover is neither able to recover the

\footnotetext{
${ }^{2} \mathrm{~A}$ minimum interval of $2 T$ can be shown to be sufficient.

${ }^{3}$ Thus, again with reference to Fig. 1, the elliptical field of view is actually the combination of the antenna pattern and of the maximum transmitting distance of the signal emitted by the sensors.

${ }^{4}$ Actually, the depicted times should be $\tau_{i}+k T$ for some $k$; we write $\tau_{i}$ for simplicity; we are interested only with time differences. Note also that considering acoustic DOAs avoids possible concerns about synchronization between sensors and rover.
}

absolute times, nor it is capable of associating any pulse with its corresponding sensor position inside its field of view.

In this letter, we propose the following suboptimal approach, which is simple to understand and easy to implement. Again with reference to Fig. 2, consider the first and the last received echoes and compute $t_{m}=\left(\tau_{1}+\tau_{\mid \mathcal{F |}}\right) / 2$. Then, count how many pulses lie in the interval $\left(t_{m}-\delta / 2 v, t_{m}+\delta / 2 v\right), \delta<h$, with $\delta / v$ being the time for covering an acoustic distance $\delta$. Let $N_{s}$ be this number, where $s$ is the snapshot index; our estimation procedure is based on the observables $N_{s}$. We understand that more sophisticated strategies are possible: One might exploit more complete information (as compared to just $N_{s}$ ) contained in the received $r(t)$. Examples will be offered in [2], and we note that one such example exploits the DOA information embedded in $\tau_{|\mathcal{F}|}-\tau_{1}$.

The receiving antenna of the rover can be arbitrarily oriented, or alternatively, the rover rotates. In both cases, it may explore the whole $2 \pi$ arc, for any given position. A key assumption made here is that successive snapshots taken by the rover always involve sensors never encountered before (i.e., snapshot independence). For analysis, it is sufficient to take each sensor as having a periodic emission; for practical battery life, they would be silent unless provoked by a rover poll.

\section{DOA ESTIMATION AND PERFORMANCES}

Let $\theta \in(0, \pi)$ be the unknown DOA and $\phi_{s} \in(0,2 \pi)$ be the rover's (ellipse's) orientation at snapshot $s$. Assume that $N_{s}$, $s=1,2, \ldots$, are independent of each other, and note that this number can be approximately taken as the number of sensors that lie in the strip of width $\delta$ within the ellipse ${ }^{5}$ (see Fig. 1). For the sake of simplicity, such a region is taken as rectangular: One side is given by the ellipse's diameter (corresponding to DOA $\theta$ and rover angle $\phi_{s}$ ), and the other side is $\delta$. The area is accordingly computed as

$$
A\left(\phi_{s}, \theta\right) \approx \frac{\delta}{\sqrt{\frac{\cos ^{2}\left(\theta-\phi_{s}\right)}{h^{2}}+\frac{\sin ^{2}\left(\theta-\phi_{s}\right)}{r^{2}}}} .
$$

The basic idea behind the proposed DOA estimation procedure is that if $\theta$ is close to $\phi_{s}$, then $A\left(\phi_{s}, \theta\right)$ is small and $N_{s}$ is small as well; conversely, when $\theta$ is orthogonal to $\phi_{s}$, there is a larger area and, consequently, a larger $N_{s}$. That is, $N_{s}$ contains information about $\theta$. More precisely, $N_{s}$ is a Poisson random variable whose average value is approximately $\lambda A\left(\phi_{s}, \theta\right)$. Accordingly, the distribution of the aggregate $\left\{N_{s}\right\}_{s=1}^{M}$ of observables collected in $M$ independent snapshots is known, and from that, the $\theta$-ML (maximum likelihood) estimation can be numerically computed $^{6}$

$$
\widehat{\theta}_{M L}=\arg \max _{\theta} \prod_{s=1}^{M} \frac{\left[\lambda A\left(\phi_{s}, \theta\right)\right]^{N_{s}}}{N_{s} !} e^{-\lambda A\left(\phi_{s}, \theta\right)} .
$$

${ }^{5}$ Should $\tau_{1}$ and $\tau_{|\mathcal{F}|}$ be generated by sensors located on opposite boundaries of the rover's field of view, this would be true. Accordingly, the greater the sensor field density $\lambda$, the better the approximation works.

${ }^{6}$ We would like to stress that different antenna patterns would simply lead to different formulas for $A\left(\phi_{s}, \theta\right)$. Clearly, the proposed method is applicable to different patterns, with only some (presumably minor) numerical difference in the correspondent performances: The key is not the exact shape of the pattern but rather its eccentricity. 
As $M$ grows, the well-known asymptotic properties of ML estimation [5] become met. In particular, $E\left[\widehat{\theta}_{M L}\right] \rightarrow \theta$, and $\operatorname{VAR}\left[\hat{\theta}_{M L}\right] \rightarrow I_{M}^{-1}(\theta)$, where $I_{M}(\theta)$ is the $M$-snapshot Fisher information with respect to $\theta$. As we shortly show, numerical investigations confirm that such performances are, in practice, attained for moderately large values of $M$ (see below). Thus, computation of $I_{M}(\theta)$ is relevant and is now in order.

First, note that $I_{M}(\theta)$ is additive for independent observations-i.e., $I_{M}(\theta)=\sum_{s=1}^{M} J_{s}(\theta)$, where $J_{s}(\theta)$ is the Fisher information from snapshot $s$. Thus, defining $\xi_{s}=\lambda A\left(\phi_{s}, \theta\right)$, and using $\mathcal{P} o i(x ; \alpha)$ as a shortcut for the Poisson distribution with argument $x$ and mean $\alpha$, one gets

$$
J_{s}(\theta)=E\left[\left(\frac{\partial \xi_{s}}{\partial \theta} \frac{\partial}{\partial \xi_{s}} \ln \mathcal{P} \operatorname{oi}\left(N_{s} ; \xi_{s}\right)\right)^{2}\right]=\left(\frac{\partial \xi_{s}}{\partial \theta}\right)^{2} \frac{1}{\xi_{s}} .
$$

The following approximation is justified by a standard Monte Carlo integration approach, amounting to replacing the arithmetic mean by the statistical expectation with respect to $\phi_{s}$, this latter assumed uniformly distributed in $(0,2 \pi)$ :

$$
\begin{aligned}
I_{M}(\theta) & =\sum_{s=1}^{M} J_{s}(\theta) \underset{\approx}{\operatorname{large} M} \frac{M}{2 \pi} \int_{0}^{2 \pi} J_{s}(\theta) d \phi_{s} \\
& =\frac{M \lambda}{2 \pi} \int_{0}^{2 \pi}\left(\frac{\partial}{\partial \theta} A\left(\phi_{s}, \theta\right)\right)^{2} \frac{1}{A\left(\phi_{s}, \theta\right)} d \phi_{s} \\
& =M \lambda \delta r \mathcal{G}\left(\frac{h}{r}\right)=M N_{e f f} \mathcal{G}(a) .
\end{aligned}
$$

In the last equality, we have defined $N_{\text {eff }}=\lambda \delta r$, and the ellipse's aspect ratio $h / r$ has been denoted as $a$. The function $\mathcal{G}(a)$ is expressible in terms of complete elliptic integrals of the first and second kind, $\mathcal{K}(a)$ and $\mathcal{E}(a)$, respectively (see [1, form. 17.3.1, 17.3.3] for the definitions). In fact

$$
\mathcal{G}(a)=\frac{2}{3 \pi}\left[\left(1+a^{2}\right) \mathcal{E}\left(1-a^{-2}\right)-2 \mathcal{K}\left(1-a^{-2}\right)\right] .
$$

From (2), we see that $I_{M}(\theta)$ is constant with $\theta$, grows linearly with $N_{\text {eff }}$ and with $M$, and further depends upon $a$.

Some comments follow.

- Asymptotically, all the $\theta$ values can be estimated with the same accuracy.

- $\delta r$ represents the effective area of the visible region; that is to say, it is the larger area available for the clustered sensors' counting process. Accordingly, $\lambda \delta r$ is the average number of sensors inside such region (the effective number $N_{\text {eff }}$ ).

- $\mathcal{G}(a)$ decreases in $a$, implying that the more eccentric the rover's field of view (ellipse), the more effective the estimation of the DOA.

- Ideally, for a prescribed $\lambda$, one would have $h / r \rightarrow 0$, with the product $\delta r$ held fixed. However, $r$ cannot increase without bound for obvious reasons ( $r$ is proportional to the maximum transmitting distance of sensors), nor can we have $h<\delta$; that is, $N_{\text {eff }}$ and $a$ cannot be assigned independent of each other.

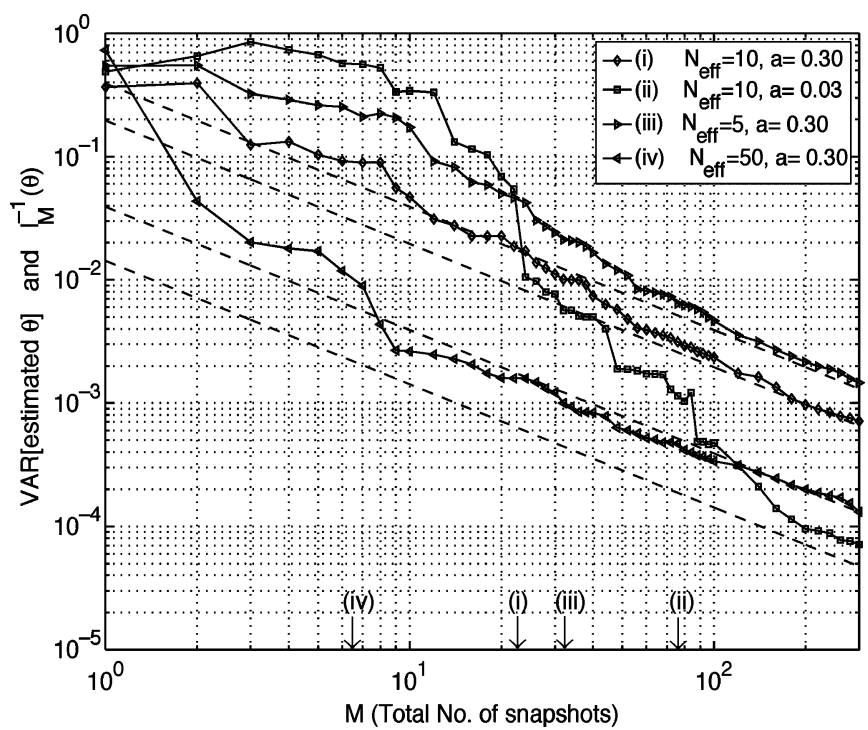

Fig. 3. Variance of $\widehat{\theta}_{M L}$ compared to the inverse of Fisher information versus the total number of snapshots taken $M$. Four combinations of the relevant parameters are addressed. The arrows on the horizontal axis denote the points after which bias in the estimate becomes negligible, i.e., $E\left[\widehat{\theta}_{M L}\right] \approx \theta$.

- In the limit as $a \rightarrow 1$

$$
I_{M}(\theta) \sim \frac{1}{2} M N_{\mathrm{eff}}(1-a)^{2}
$$

and the opposite extreme of $a \rightarrow 0$

$$
I_{M}(\theta) \sim \frac{2}{3 \pi} M N_{\mathrm{eff}} a^{-1} .
$$

The Fisher proxy is reasonable, provided that $\widehat{\theta}_{M L}$ attains its large-sample optimality: $E\left[\widehat{\theta}_{M L}\right] \rightarrow \theta$ and $\operatorname{VAR}\left[\widehat{\theta}_{M L}\right] \rightarrow$ $I_{M}^{-1}(\theta)$. Also, recall that there are approximations in the proposed model: One is the way we computed the area in (1).

We have simulated, with the double aim of checking the approximations and of investigating at what values of $M$ the asymptotic performances seem to be attained. In the simulations, an ellipsoidal field of view is used, for simplicity and to correspond to the explicit bounds. In Fig. 3, the variance of the estimator $\widehat{\theta}_{M L}$ is compared to the inverse of Fisher information, as given in (2). We see that within a reasonable number of snapshots, the asymptotic performances are met. ${ }^{7}$ The down-arrows on the horizontal axis denote the point after which the absolute value of the estimator bias stays below $10^{-2}$. To check the approximations, for given $N_{\text {eff }}$ and $a$, we have run simulations using different combinations of the relevant parameters $\lambda, r, h$, and $\delta$. Qualitatively, the results are close to those given in Fig. 3. For instance, in this way, we have verified that the speed of convergence of the variance to its asymptote is essentially insensitive to the ratio $\delta / h$ : In Fig. 3, we have chosen $\delta / h=1 / 3$, but doubling this value basically yields the same results. ${ }^{8}$ In summary, the simulations corroborate the analysis and validate the analytical relationships.

\footnotetext{
${ }^{7}$ In judging the practical impact of $M$, recall that the number of different rover locations is just $M$ divided by the number of snapshots taken in a fixed position

${ }^{8}$ Clearly, for $\delta \approx h$, the analytical approximation behind (1) fails.
} 


\section{SUMMARY}

We have investigated the DOA estimation by a network of isotropic sensors polled by a travelling "rover," with the system design based upon the SENMA paradigm. The novelty is that the sensors are unusually "dumb" in that they have individually no DOA capability; indeed, they have no capabilities at all except that of emitting a periodic signal following their encounter with the wavefront whose DOA is sought. The sensors are inexpensive and randomly located, they do not communicate with each other, and their positions are unknown both to them and to the rover.

The idea is that they send a periodic train of short pulses that starts at the time instant that the sensor is hit by an acoustic wave of short duration. (Actually, the physical transmission to the rover is virtual until the rover polls the sensor.) The key point is that the rover's field of view is eccentric (taken here as elliptical, but that is only for ease of analysis). The DOA information is contained in the number of sensors within a "stripe" in the rover's field of view and oriented orthogonal to the DOA. This number is taken as Poisson distributed. The results are remarkably good, and asymptotically efficient performance is obtained with a reasonable number of snapshots taken by the rover.

\section{REFERENCES}

[1] M. Abramowitz and I. A. Stegun, Handbook of Mathematical Functions. New York: Dover, 1964.

[2] S. Marano, V. Matta, P. Willett, and L. Tong, "Support-based and ML approaches to DOA estimation in a dumb sensor network," IEEE Trans. Signal Process., submitted for publication.

[3] P. Willett and L. Tong, "One aspect to cross-layer design in sensor networks," in Proc. MILCOM, Monterrey, CA, Oct. 2004, pp. 688-693.

[4] L. Tong, Q. Zhao, and S. Adireddy, "Sensor networks with mobile agents," in Proc. MILCOM, Boston, MA, Oct. 2003.

[5] H. L. Van Trees, Detection, Estimation and Modulation Theory. New York: Wiley, 1968, pt. 1. 